\title{
Modelado de la Producción de Biomasa Microalgal en un Fotobiorreactor de Burbujeo Anular y Configuración Variable
}

\author{
Enrique J. Rost, Susana G. Perales, María Rosa Carstens y Laura B. Pérez \\ Universidad Nacional de la Patagonia San Juan Bosco, Facultad de Ingeniería y Facultad de Ciencias \\ Naturales, Ruta Prov. 1 Km 4, 9000 Comodoro Rivadavia, Chubut, Argentina. (e-mail: erost@unpata.edu.ar, \\ peralessusana2@gmail.com,marisa@unpata.edu.ar, perezlulib@yahoo.com.ar).
}

Recibido Jul. 28, 2016; Aceptado Sep. 30, 2016; Versión final Nov. 30, 2016, Publicado Abr. 2017

\begin{abstract}
Resumen
Se presenta el modelado de los datos empíricos obtenidos en el cultivo de microalgas marinas (Phaeodactylum tricornutum y Tetraselmis sp.) en un fotobiorreactor de escala piloto de tubos concéntricos verticales con burbujeo en el espacio anular. El reactor empleado permite alternar entre dos configuraciones que involucran diferentes volúmenes y modelos de flujo. Se ha podido concluir acerca del efecto que sobre la producción de biomasa provoca un aumento del volumen de cultivo en desmedro de la captación de energía lumínica. Para considerar el efecto de ensombrecimiento de células a media y alta concentración sobre la velocidad de crecimiento se propone un modelo con afectación simple de la ecuación cinética de reproducción exponencial.
\end{abstract}

Palabras clave: producción de biomasa, microalgas, fotobiorreactor, modelado, ensombrecimiento mutuo

\section{Modeling of Microalgae Biomass Production in a Variable Configuration Annular Bubbling Photobioreactor}

\begin{abstract}
The results of the modeling of empirical data relative to marine microalgae cultures (Phaedactylum tricornutum and Tetraselmis sp.) in a pilot photobioreactor of two vertical concentric tubes with bubbling in the annular space, are presented. The geometry of this reactor switches between two configurations involving different culture volume and different hydrodynamics. The effect on biomass productivity that provokes an increasing in volume of culture at the expense of diminishing the capture of light energy has been analyzed. Finally, to consider the influence of shadowing of the cells on the biomass increment at medium and high concentration, a model is proposed with involvement of the exponential growth kinetic equation.
\end{abstract}




\section{INTRODUCCION}

Entre las variantes más empleadas de fotobiorreactores (FBR) para el cultivo de microalgas con fines experimentales e industriales se encuentran las de tubos cilíndricos verticales, con un sistema de burbujeo en su base para la introducción de un determinado caudal de aire y una fracción menor de $\mathrm{CO}_{2}$ (Xu et al., 2009; Pawar, 2016). Las mismas constituyen una alternativa ventajosa debido a su geometría compacta, de bajo costo y facilidad de operación. El burbujeo impacta de manera ventajosa sobre la producción de biomasa al crear en el medio de cultivo una turbulencia que mejora la transferencia de gases y provoca que el total de las células se trasladen a través de la zona de mayor incidencia lumínica con alta frecuencia. Entre las geometrías de cilindros verticales más estudiadas se encuentran las de un cilindro vertical simple (bubble column) y de tubos concéntricos (draft column), en ambos casos con un burbujeador central y caracterizados por un flujo ascendente en el centro del reactor y descendente por la zona inmediata a la pared externa. A éstas se agrega la configuración de tubo dividido (split column), con un tabique plano vertical que separa el espacio interior en dos mitades y con burbujeador desplazado hacia una de éstas.

La representación mediante modelos matemáticos del comportamiento de un sistema del tipo tratado aquí se dificulta notoriamente debido, principalmente, a la interacción de los efectos fluidodinámicos y de fotosíntesis de las células. Se han presentado modelados de la evolución de la biomasa sobre consideraciones geométricas y fluidodinámicas diversas para beneficio del diseño y el escalamiento de las unidades de producción (Sánchez Mirón et al., 2002, Luo et al., 2004, 2012, Wu y Merchuk, 2004, Pruvost et al., 2006, Pawar, 2016) y considerando el análisis del aporte de diversos parámetros que afectan el proceso de reproducción celular. Entre éstos se tienen, por su mayor influencia, los inherentes a la magnitud de la energía lumínica captada por la biomasa (Barbosa et al., 2003, García-Malea López et al., 2006, Merchuk et al., 2011), la relación área iluminada a volumen de cultivo (Mirón et al., 1999, Joshi et al., 2001, Xu et al., 2009), la transferencia y el secuestro de $\mathrm{CO}_{2}$ (Kumar y Das, 2012, Ying et al., 2013, Kong y Vigil, 2014, Hosseini et al., 2015) e incluso el estrés celular de origen hidromecánico (Michels et al., 2010, López Rosales et al., 2015), los dos últimos afectados por detalles como el tamaño de burbujas y la velocidad superficial del gas (Vial et al., 2002, Barbosa et al., 2004).

Por su lado, los modelos matemáticos de fotosíntesis desarrollados para el comportamiento del sistema bioquímico se han basado en la síntesis de una gran cantidad de etapas reales o hipotéticas que han sumado notoria dificultad a la tarea de resolución de los mismos. De modo que para no hacer tan ardua la tarea computacional al momento de simular el comportamiento de un FBR, sobre todo para su utilización en cálculos de ingeniería, se conviene en adoptar modelos cinéticos simples. Siendo que la irradiancia es la principal variable que afecta la fotosíntesis (asegurada la disponibilidad de nutrientes necesarios para los procesos bioquímicos), se ha encontrado ventajoso a estos efectos realizar la predicción de la velocidad de crecimiento de la biomasa sólo en función de este parámetro, encontrándose propuestas variadas en la bibliografía científica (Merchuk et al., 2011).

El principal fenómeno que afecta la captación lumínica por parte de la biomasa es que las células que se encuentran más cercanas a la pared iluminada del FBR provocan que la luz llegue progresivamente atenuada a las que se encuentran en el interior del recipiente, efecto que se intensifica con el aumento de la concentración. Es entonces cuando el modelo de reproducción exponencial deja de ser efectivo y el cultivo ingresa a una etapa de velocidad de crecimiento estacionaria o lineal. El análisis del diseño de un fotobiorreactor introduciendo las consideraciones pertinentes al fenómeno de atenuación fue original y extensivamente estudiado por Bernárdez et al. (1987). A su vez, la transmisión de luz en el medio de cultivo ha sido convenientemente caracterizada por modelos basados en la ley de Lambert-Beer tomando en consideración la geometría de un FBR tubular (Evers, 1991; Barbosa et al., 2003, García-Malea López et al., 2006, Wu y Merchuk, 2001, 2004, Merchuk et al., 2011).

En este trabajo se presentan los resultados obtenidos con un FBR de escala piloto con una geometría de tubos concéntricos con burbujeo en el espacio anular entre tubos, lo que permite disponer de dos variantes de volumen e hidrodinámica para estudiar el efecto de un mayor volumen de producción en desmedro de una menor captación de energía lumínica. Sobre consideraciones simplificatorias basadas en la configuración de la unidad experimental se propone luego un modelado del crecimiento de la biomasa en sus fases exponencial y lineal, introduciendo el efecto de atenuación en las etapas de media y alta concentración mediante una variante simplificada de la cinética propuesta previamente por Molina Grima et al. (1994), obteniéndose resultados estadísticamente satisfactorios.

\section{MATERIALES Y METODOS}

El FBR experimental que se dispuso para este trabajo es de tubos concéntricos con burbujeo en el espacio anular. De acuerdo a los esquemas presentados en la figura 1, el mismo permite trabajar con dos configuraciones alternativas de interés para la obtención de datos empíricos: a) de flujo restringido, con el cultivo desarrollándose en el espacio anular entre tubos (el tubo interno permanece vacío) y b) con flujo total, donde el cultivo además del volumen anular ocupa el interior del tubo interno y por efecto air-lift se obtiene un flujo ascendente por el espacio anular con retorno o flujo descendente por el tubo interno. 
Los tubos son de metacrilato de etilo con las siguientes dimensiones: cilindro externo de 200 mm de diámetro externo (pared de $4 \mathrm{~mm}$ ) y cilindro interior de $120 \mathrm{~mm}$ (pared de $4 \mathrm{~mm}$ ), con una longitud total o altura de 1000 $\mathrm{mm}$. En la variante de flujo total la longitud del tubo interno es de $750 \mathrm{~mm}$, ubicándose su extremo inferior a $20 \mathrm{~mm}$ por encima del fondo del FBR. De este modo, la capacidad de cultivo de la unidad es de $15 \mathrm{~L}$ en flujo restringido y $22 \mathrm{~L}$ en flujo total.

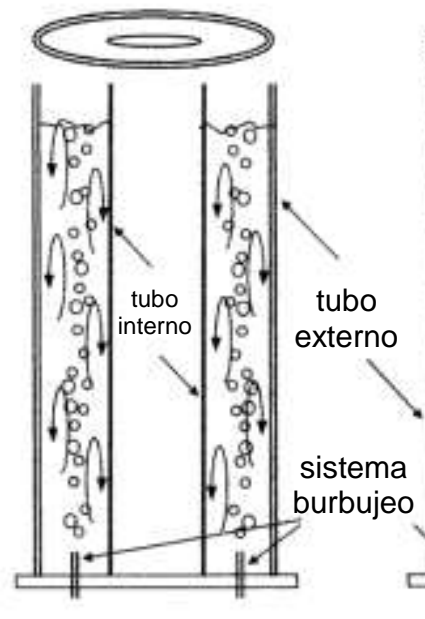

a) flujo restringido

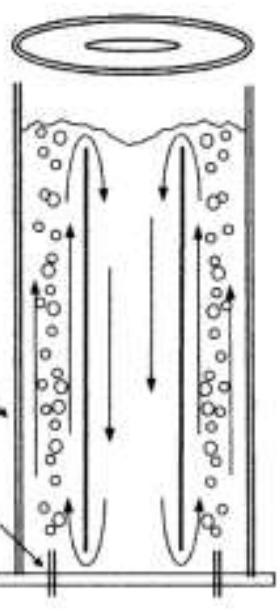

b) flujo total

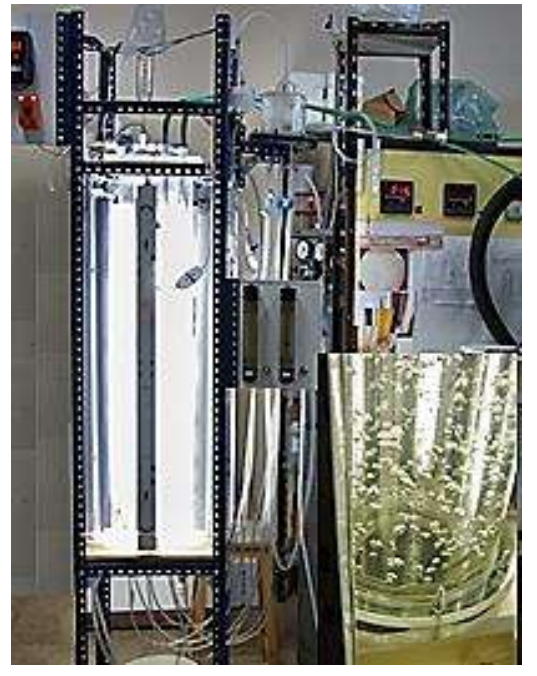

Fig. 1: Izquierda: representación esquemática de los dos modelos de flujo alternativos del FBR de burbujeo de tubos concéntricos. Derecha: imagen fotográfica de la unidad experimental con detalle del sistema de burbujeo (esquina inferior derecha).

El suministro de aire es provisto por un compresor equipado con trampa de líquidos y válvula reductora de presión y el $\mathrm{CO}_{2}$ proviene de un cilindro a presión también a través de una válvula reductora (figura 2). Los caudales de ambas corrientes son medidos mediante dos caudalímetros del tipo rotámetro, antes de mezclarse de acuerdo a la proporción que se fija mediante sendas válvulas aguja. El volumen de aire ingresado es de aproximadamente $200 \mathrm{~L} / \mathrm{h}$ y el de $\mathrm{CO}_{2}$ de $5 \mathrm{~L} / \mathrm{h}$. La mezcla se conduce luego por un filtro estéril de membrana de polietileno de tamaño de poro de 0,2 $\mu \mathrm{m}$ para eliminar contaminantes y se envía a un pulmón-distribuidor de donde la mezcla es conducida a los burbujeadores. Los gases ingresan al cultivo a través de 8 burbujeadores independientes, distribuidos simétricamente en la zona correspondiente al espacio anular, construidos con tramos horizontales y orientados circularmente de tubo de acero inoxidable de $1 / 8$ " de diámetro con tres perforaciones laterales de $1 \mathrm{~mm}$. El FBR se encuentra equipado con un sistema de luz artificial, con tubos fluorescentes de luz blanca de $30 \mathrm{~W}$ cada uno distribuidos alrededor del FBR, con una capacidad máxima de instalación de 8 tubos (lo que corresponde a una irradiancia promedio máxima disponible de $180 \mu \mathrm{E} /\left(\mathrm{m}^{2}\right.$.s $)$ en la superficie interna del FBR de acuerdo a determinaciones empíricas). Las experiencias mostradas en este trabajo se realizaron con una irradiancia promedio de $125 \mu \mathrm{E} /\left(\mathrm{m}^{2}\right.$.s $)$ y con fotoperiodo de $16 \mathrm{~h}$.

Para el medio de cultivo se ha adoptado la formulación de Laing (1991) empleando agua de mar, mantenida en oscuridad durante no menos de 4 meses y microfiltrada inmediatamente antes de su empleo (membrana de polietileno de $0,2 \mathrm{~mm}$ ). El pH del medio de cultivo se mantuvo mediante la introducción de un porcentaje constante de $\mathrm{CO}_{2}$ en la corriente de aire, normalmente entre 2 y $3 \%$ del volumen de éste (siguiendo a Wu et al., 2004, Barbosa et al., 2003), realizando periódicamente un control manual coincidente con el muestreo realizado para cuantificación del crecimiento de la biomasa.

Las cepas de microalgas cultivadas, Phaedactylum tricornutum y Tetraselmis sp. (típicas productoras de ácidos grasos omega 3) procedieron del cepario del Departamento de Biología y Ambiente de la Facultad de Ciencias Naturales, Universidad Nacional de la Patagonia San Juan Bosco, donde permanecen en medio de cultivo líquido de acuerdo a la metodología descripta por Provasoli (1968) y McLachlan (1973). Para ello se inocula $0,1 \mathrm{ml}$ de la cepa original en tubos de ensayos con $10 \mathrm{ml}$ de medio Erdschreiber líquido, que se disponen en un ambiente acondicionado a $21^{\circ} \mathrm{C}$ con iluminación intermitente (fotoperiodo de $16 \mathrm{hs)}$ con una intensidad lumínica de $120 \mu \mathrm{E} /\left(\mathrm{m}^{2}\right.$.s). Los repiques se realizan cada 25 días. La preparación del inóculo que se incorpora en el FBR se realiza a partir del cultivo en volumen de entre 125 a $500 \mathrm{~cm}^{3}$, con un medio de cultivo idéntico al que se emplea en la unidad piloto. En el proceso de cambio de volumen se emplea una concentración inicial de $5 \times 10^{4} \mathrm{cel} / \mathrm{ml}$, en condiciones de crecimiento en fase de adaptación. En la operación del FBR piloto y entre ciclos de cultivos, se inició en la mayoría de los casos con una concentración de biomasa superior a 0,05 g/L (en base seca) para promover el inicio del cultivo habiendo superado la fase de adaptación, a los mejores efectos del presente análisis. 


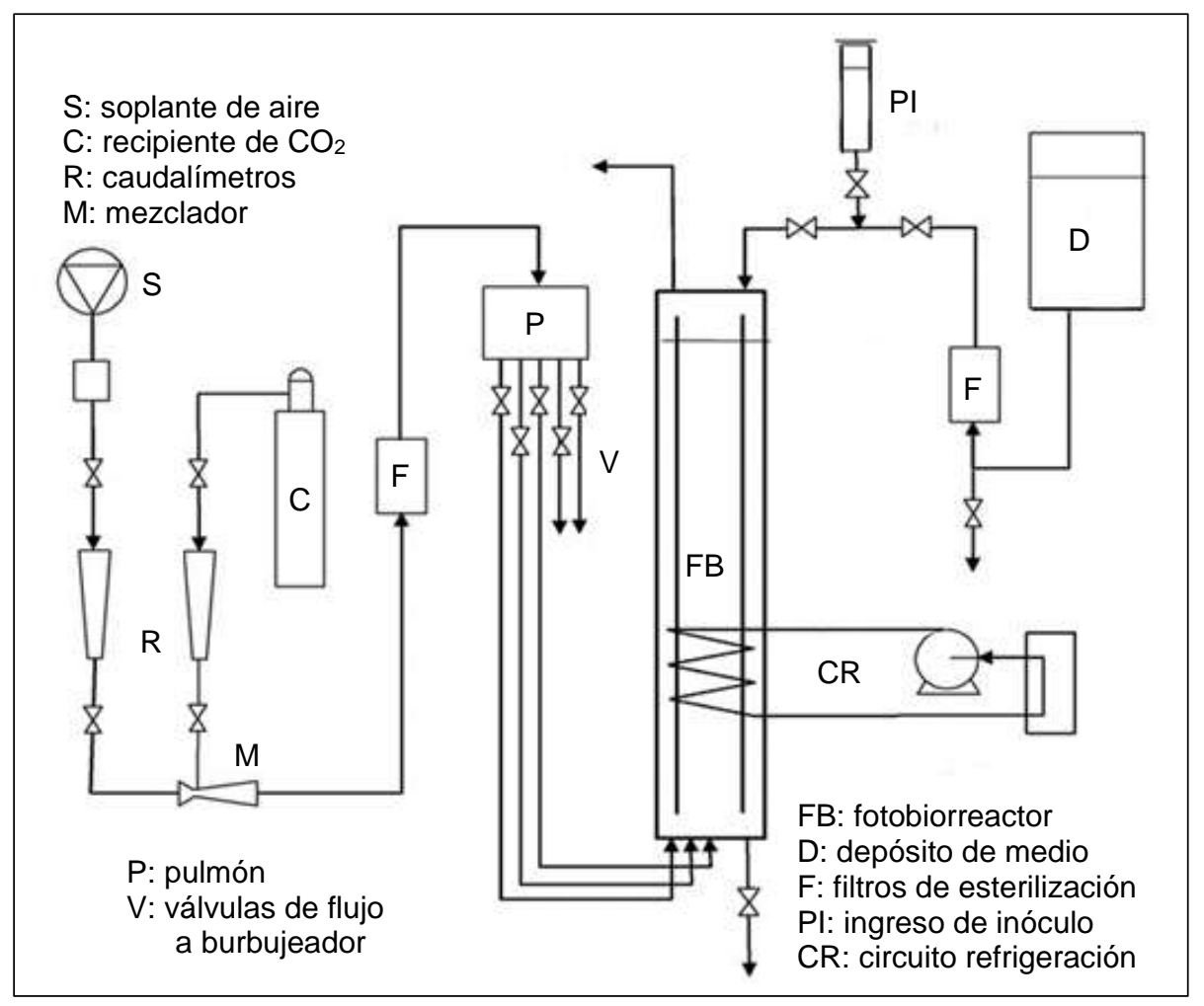

Fig. 2: Esquema de la instalación experimental con indicación de los elementos principales.

EI FBR fue operado en modo semicontinuo, esto es, realizando cosechas parciales en períodos de crecimiento en fase lineal. El período de cultivo entre cosechas se fijó determinando en el primer ciclo el cambio de fase lineal a estacionaria, de modo de estimar la concentración de biomasa máxima alcanzable. A los efectos de no introducir disturbios en el cultivo, el muestreo periódico se realizó extrayendo cantidades mínimas, del orden de $10 \mathrm{~mL}$, determinando la concentración de biomasa por técnica turbidimétrica mediante un fotómetro Hach modelo DR2010. El factor de respuesta de este instrumento se ajustó tomando de referencia la concentración de biomasa en la finalización de cada ciclo. Al mismo tiempo se realizaron las mediciones de $\mathrm{pH}$. Y la determinación de la concentración final de cada ciclo se realizó tomando muestras de $400 \mathrm{~mL}$ del cultivo cosechado, separando la biomasa por centrifugación y procediendo a secar la misma en estufa a $50^{\circ} \mathrm{C}$ hasta obtener peso constante.

\section{Modelado}

Durante el proceso de reproducción de las microalgas, en forma semejante a otros microorganismos, ocurren etapas características que es necesario describir para lograr un adecuado modelado del mismo. En condiciones de ausencia de limitación de insumos, cuando la concentración de biomasa es muy pequeña, se presenta una etapa inicial de adaptación seguida de una fase de rápido crecimiento de orden exponencial. Se presenta luego una situación de competencia espacial en la captura de la energía lumínica disponible para fotosíntesis, debido a que las células más cercanas a la superficie externa del FBR interceptan la luz ensombreciendo a las restantes (efecto de atenuación celular de la irradiancia). Entonces se puede observar que el incremento de la concentración de biomasa abandona el modelo exponencial y se ajusta a un modelo lineal o de productividad constante. Este período es notoriamente largo y se mantiene hasta que, debido a limitación de los recursos de consumo (tanto lumínicos como de nutrientes), ocurre una rápida disminución de la tasa de crecimiento y se ingresa en una fase de concentración estacionaria con equilibrio entre las velocidades de reproducción y de muerte de las células (con productividad nula). Finalmente, si se mantiene el cultivo con una tasa de muerte superior a la de reproducción, se produce una fase de declive (tasa negativa de crecimiento) con tendencia a desaparición de la biomasa. A los efectos de la producción de biomasa el periodo de crecimiento lineal es de suma importancia pues además de ser fácilmente controlable ofrece la máxima velocidad de crecimiento real.

En la etapa de crecimiento exponencial típicamente la velocidad de producción se ajusta al modelo cinético:

$$
\frac{1}{\mathrm{C}} \frac{\mathrm{dC}}{\mathrm{dt}}=\mathrm{m}_{\max }
$$


en el que $C$ es la concentración másica y $\mu$ max la velocidad específica máxima. Esta ecuación puede integrarse, en condiciones de cultivo discontinuo y con concentración inicial Co para obtener la función:

(4) $\mathrm{C}=\exp (\mathbf{t})_{\max }$

Dado que la velocidad de reproducción se ve afectada por el fenómeno de ensombrecimiento mutuo de las células, el modelo exponencial sólo es útil para concentraciones de biomasa reducidas. Para simular la fase de crecimiento no exponencial o lineal, se ha encontrado aquí que es posible mantener el modelo anterior afectando la velocidad específica por el cociente de la irradiancia promedio en el medio de cultivo lav y la irradiancia de la luz incidente en la superficie externa lo de la forma:

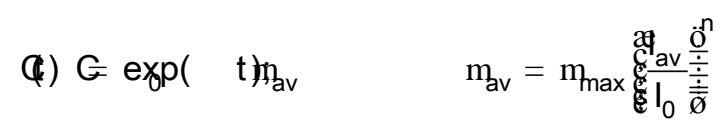

Esta cinética con exponente $\mathrm{n}$ del cociente de irradiancias constituye una simplificación del empleado previamente por Molina Grima et al. (1994, 1999). La irradiancia promedio es función de la concentración instantánea. Para evaluar la irradiancia o densidad de flujo fotónico en el interior de un FBR se han desarrollado ecuaciones basadas en la ley de Lambert-Beer. Aunque válida sólo para propagación unidimensional, es considerada satisfactoria para su aplicación a un FBR cilíndrico sobre la base de considerar que la superficie del mismo está uniformemente iluminada y que cada punto de la superficie interna de la pared se comporta como una fuente de luz. Entonces, la irradiancia en el interior del medio de cultivo puede obtenerse integrando sobre la superficie de incidencia mediante la ecuación (Evers, 1991):

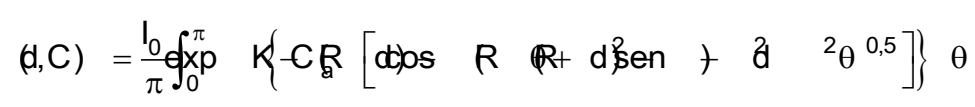

donde Ka es el coeficiente de extinción, $R$ el radio del cilindro del FBR y d la distancia desde la superficie del reactor al elemento receptor (en esta ecuación no se considera la interferencia de la pared del FBR).

Cuando la concentración de biomasa es alta la I(d,C) es sustancial sólo cerca de la superficie del FBR y en esta situación la ecuación (4) se puede reducir a (Barbosa et al., 2003, García-Malea López et al., 2006, Wu y Merchuk, 2001, 2004):

$$
\text { (d,C) } I=\exp \left(K \quad \text { a } C_{2}\right)
$$

Finalmente, la irradiancia promedio en el interior del cultivo se puede calcular integrando la función previa, obteniéndose la siguiente ecuación (Acién et al., 1998, García-Malea López et al., 2006):

$$
\mathrm{I}_{\mathrm{av}}=\frac{\mathrm{I}_{0}}{\mathrm{~K}_{\mathrm{a}} \mathrm{RC}}[1-\exp (\mathrm{K} \mathrm{RC}]
$$

A los efectos de la aplicación del modelo de acuerdo a la geometría del FBR empleado aquí, se ha identificado a $\mathrm{R}$ con la profundidad del cultivo en el espacio anular, o sea el espesor de este volumen, lo cual resulta apropiado operando con el FBR en la variante de flujo restringido. No obstante, en el caso de flujo total se ha hecho la misma consideración dado que, como la irradiancia I $(\mathrm{d}, \mathrm{C})$ disminuye muy rápidamente en las capas líquidas más cercanas a la pared iluminada, en el volumen del tubo interno se puede despreciar la captación de energía lumínica por parte de las células, lo cual predomina en la medida que su concentración avanza con el cultivo. Finalmente, se consideró aceptable emplear el valor de irradiancia promedio en el espacio anular debido a que el intenso mezclado provocado por el burbujeo de gases permite inferir que todas las células acceden a dosis de energía lumínica en forma homogénea en su recorrido por esta zona.

\section{RESULTADOS}

En las figuras 3 y 4 se presentan las evoluciones de concentración de biomasa determinadas mediante el cultivo de $P$. tricornutum y Tetraselmis $s p$. respectivamente, correspondiendo en cada caso el diagrama de la izquierda a la operación del FBR en configuración de flujo restringido (volumen de cultivo de $15 \mathrm{~L}$ ) y el diagrama de la derecha en la configuración de flujo total (22 L), con 3 ciclos o cosechas cada uno (operación semicontinua). Las velocidades de crecimiento en fase lineal obtenidas mediante el modelo cinético ajustado y de acuerdo a datos empíricos son las indicadas en la tabla 1.

Para representar matemáticamente la evolución de la concentración de biomasa en cada ciclo, de acuerdo a lo indicado en Modelado, se empleó el modelo de crecimiento exponencial modificado, mediante la resolución simultánea de las ecuaciones (3) y (6). A estos efectos, no se incluyeron en los cálculos los datos empíricos que se consideraron anteriores al período exponencial (con concentraciones por debajo de $0,05 \mathrm{~g} / \mathrm{L}$ ) y posteriores al desarrollo de la fase lineal (cuando se hace notorio el decaimiento de la velocidad de crecimiento). 
Consecuentemente, los datos de concentración iniciales presentados en la figura 3 (tiempo 0 para cada ciclo) se descartaron para el ajuste del modelo matemático por corresponder a la etapa de adaptación y también se descartaron los datos finales de los 3 ciclos del cultivo de $P$. tricornutum con flujo total por considerarse posteriores a la etapa de crecimiento lineal. Por su lado, los datos de concentración inicial y final del primer ciclo de la experiencia en flujo restringido presentados en la figura 4 (cultivo de Tetraselmis sp.) se descartaron también por corresponder a las etapas previa y posterior a las de estudio.
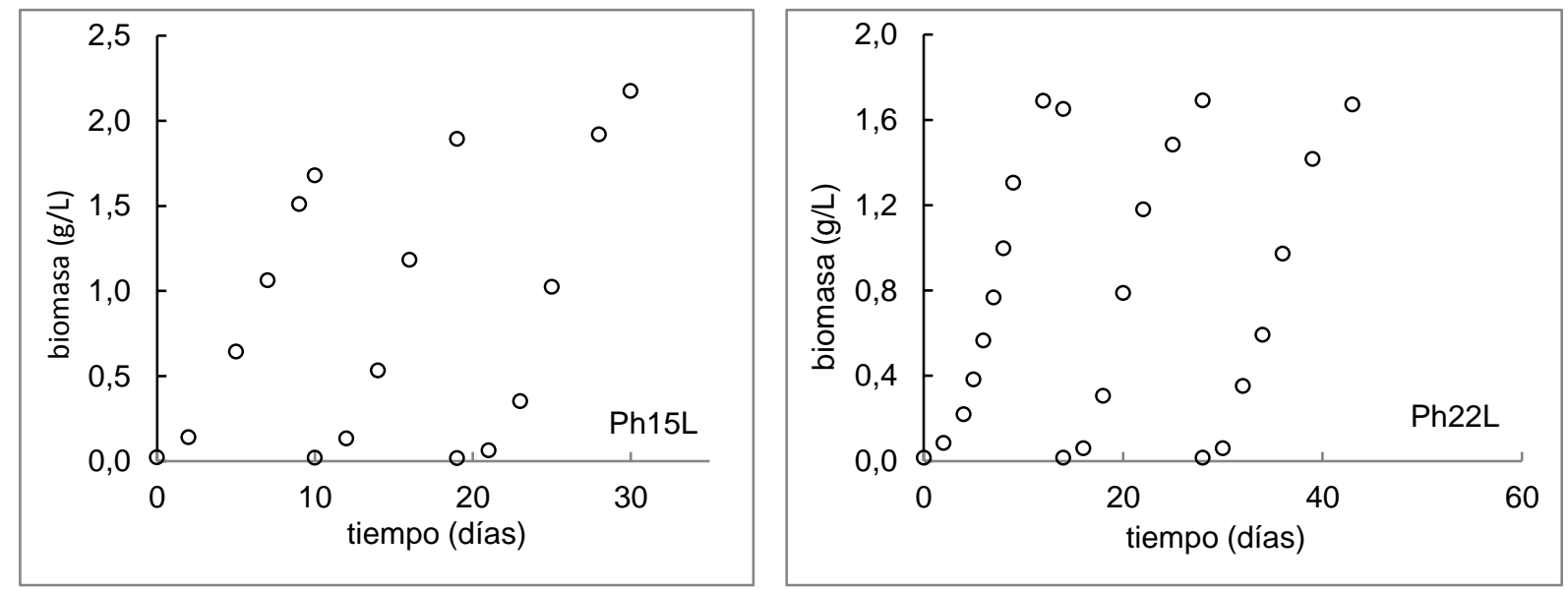

Fig. 3: Evolución de la concentración de biomasa en experiencias realizadas con $P$. tricornutum. El diagrama de la izquierda corresponde a operación con el FBR en flujo restringido ( $15 \mathrm{~L}, 3$ ciclos) y el de la derecha a operación en flujo total (22 L, 3 ciclos).
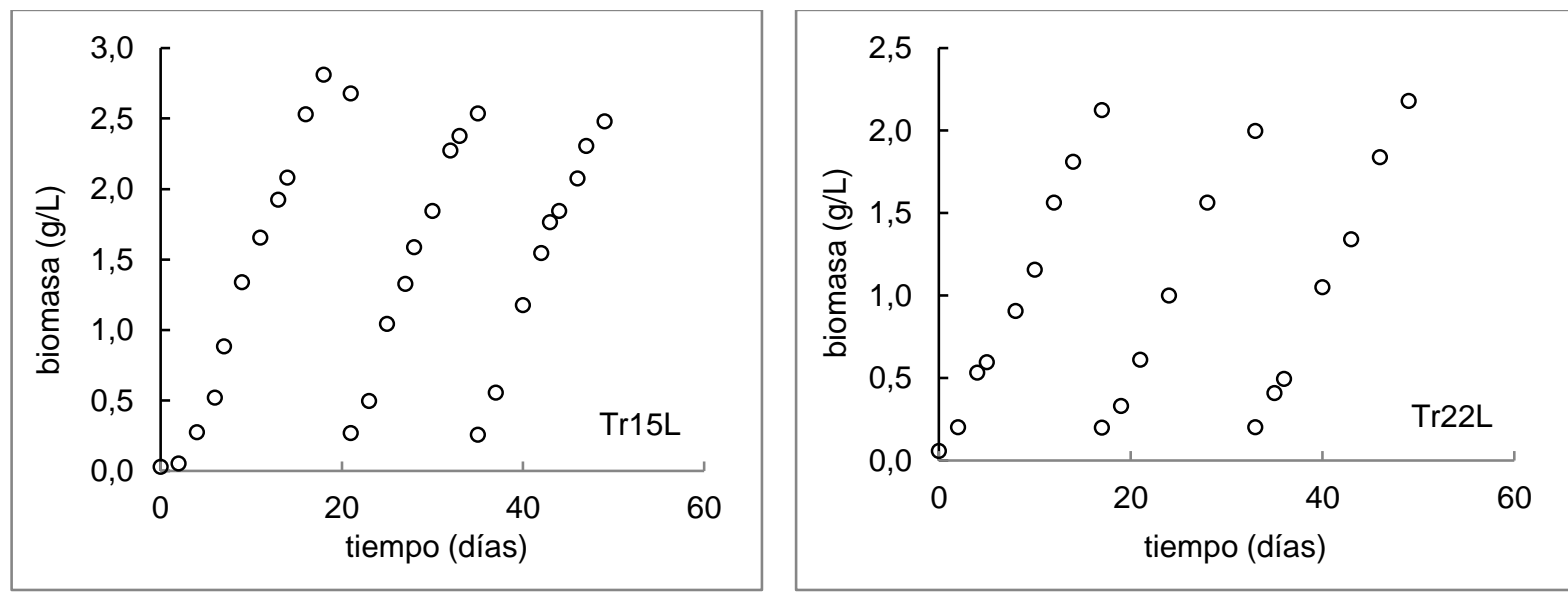

Fig. 4: Evolución de la concentración de biomasa en experiencias realizadas con Tetraselmis sp. El diagrama de la izquierda corresponde a operación con el FBR en flujo restringido (15 L, 3 ciclos) y el de la derecha a operación en flujo total (22 L, 3 ciclos).

Las figuras 5 y 6 presentan gráficamente los resultados de ajuste obtenidos mediante el modelo adoptado (línea llena), incluyendo para comparación la curva de crecimiento exponencial (línea a trazos). Los valores empíricos se indican mediante marcadores (círculos). En el diagrama de la derecha de la figura 5 se incluyeron los 3 valores de concentración finales del cultivo con $P$. tricornutum en flujo total que fueron descartados de acuerdo a lo indicado previamente para el tratamiento estadístico, a los efectos de mostrar su desviación respecto del comportamiento en fase lineal.

El coeficiente de extinción $\mathrm{K}_{\mathrm{a}}$ fue determinado para las dos especies mediante medidas fotométricas de absorbancia en $540 \mathrm{~nm}$ en función de concentración de biomasa $C$ en una celda de longitud $L$ de $25 \mathrm{~mm}$ y de acuerdo a la siguiente relación (Molina Grima et al., 1997):

$$
\mathrm{Ka}=\mathrm{A} 540 /(\mathrm{C} L)
$$

Los valores promedio obtenidos fueron de $\mathrm{Ka}=0,11$ y $0,12 \mathrm{dm}^{2} / \mathrm{g}$ para $P$. tricornutum y Tetraselmis sp., respectivamente. 

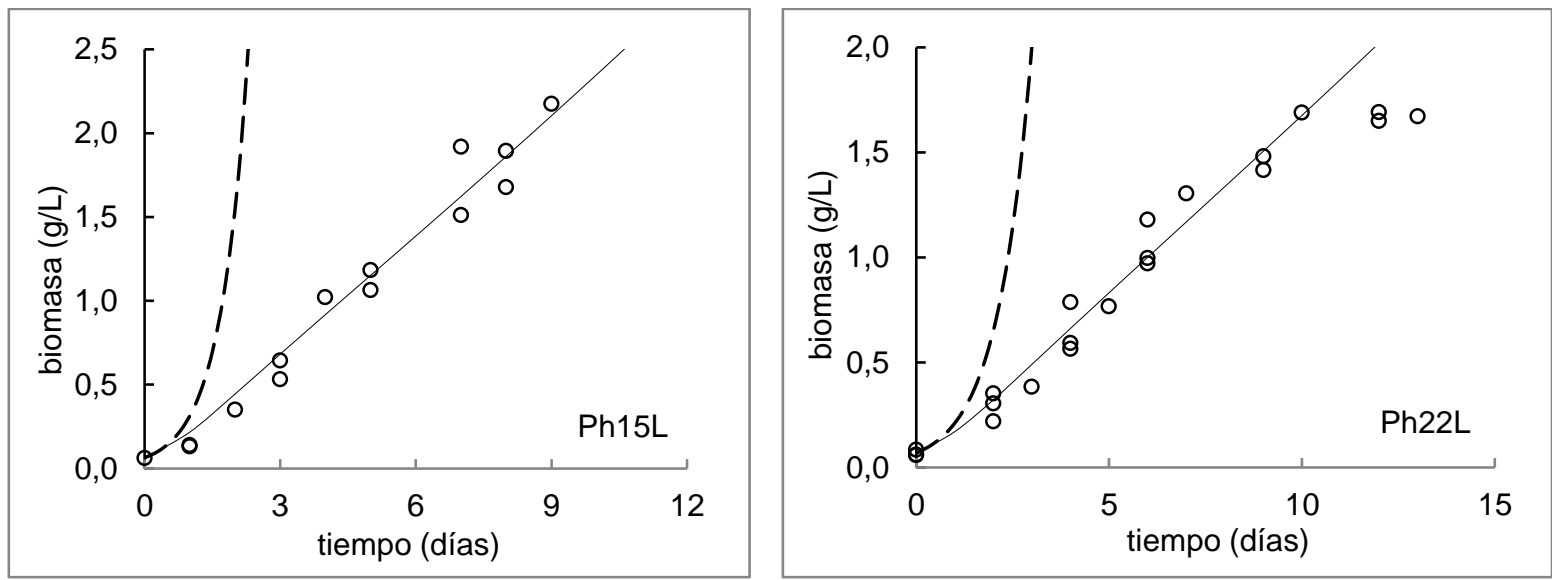

Fig. 5. Incremento de la concentración de biomasa con $P$. tricornutumen flujo restringido (15 L, diagrama de la izquierda) y en flujo total (22 L, derecha) (conjunto de datos de 3 ciclos).
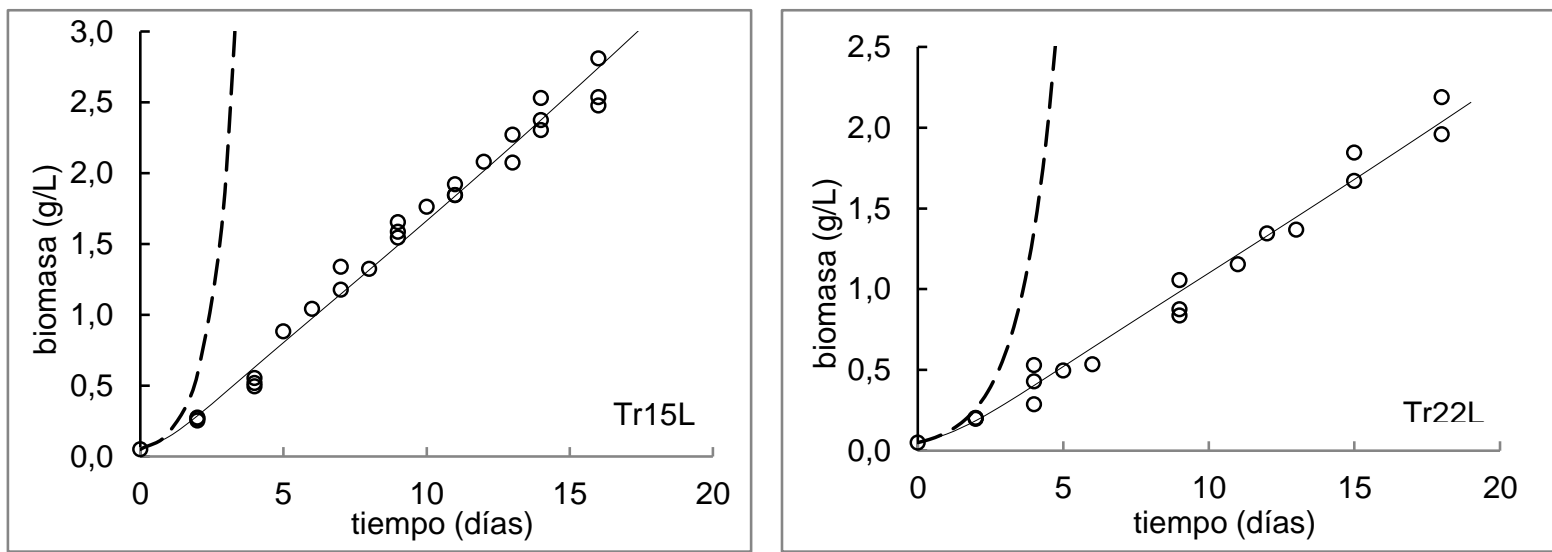

Fig. 6: Incremento de la concentración de biomasa con Tetraselmis sp. en flujo restringido (15 L, diagrama de la izquierda) y flujo total ( $22 \mathrm{~L}$, derecha) (conjunto de datos de 3 ciclos).

Los valores de los parámetros de simulación ajustados y los valores finales de productividad de acuerdo al modelo se presentan en la tabla 1. Los valores de la velocidad específica máxima $\mu_{\max }$ indicados para ambas especies en las dos configuraciones ensayadas, así como el del exponente $n$ de la relación entre irradiancias promedio e incidente, se obtuvieron mediante ajuste por mínimos cuadrados de las diferencias de concentración de biomasa entre valores empíricos y del modelo propuesto. Dentro de este esquema estadístico se estableció que el el exponente $n$ debía mantener su valor independientemente de la variación de configuración del FBR y de la especie biológica que se ensaye. El mejor ajuste para las experiencias informadas se obtuvo con $n=0,675$ (los valores de $n$ para el mejor ajuste individual de las 4 experiencias tuvieron un promedio de $n=0,643$ con una desviación estándar de 0,069).

La tabla 1 muestra también los valores ajustados del exponente de la velocidad específica máxima, de productividad y de producción de biomasa en fase lineal para las 4 experiencias de cultivo aquí informadas. En la última columna se indica la productividad calculada con datos empíricos de concentración promedio. A los efectos del cálculo de productividad y producción en cada una de las 4 experiencias, se tomaron datos del modelo y empíricos por encima de $0,500 \mathrm{~g} / \mathrm{L}$, considerada esta condición suficiente para encontrarse el cultivo plenamente en su fase de crecimiento lineal (o de máxima velocidad de crecimiento de la biomasa).

Tabla 1: Valores ajustados del exponente $n$, de la velocidad específica máxima $\mu_{\max }$, de producción y de productividad de biomasa en fase lineal según modelo y datos experimentales.

\begin{tabular}{|c|c|c|c|c|c|}
\hline & $\begin{array}{c}n \\
-\end{array}$ & $\begin{array}{c}\mu_{\max } \\
\text { día }^{-1}\end{array}$ & $\begin{array}{c}\text { Producción } \\
\text { g/día }\end{array}$ & $\begin{array}{c}\text { Productividad } \\
g /(L . \text { día })\end{array}$ & $\begin{array}{c}\text { Produc. Exper. } \\
g /(L . \text { día })\end{array}$ \\
\hline Ph15L & 0,675 & 1,593 & 3,538 & 0,236 & 0.240 \\
\hline Ph22L & 0,675 & 1.126 & 3,711 & 0,169 & 0.160 \\
\hline Tr15L & 0,675 & 1,229 & 2,573 & 0,172 & 0,171 \\
\hline Tr22L & 0,675 & 0,827 & 2,549 & 0,116 & 0,110 \\
\hline
\end{tabular}




\section{CONCLUSIONES}

En las experiencias presentadas de cultivo de microalgas en un FBR de cilindros concéntricos con burbujeo en el espacio anular, de escala piloto de $15 \mathrm{~L}$ de capacidad en flujo restringido y $22 \mathrm{~L}$ en flujo total, se registraron períodos de incremento lineal sostenido de las concentraciones de biomasa para concentraciones medias y altas. Tanto la velocidad de reproducción de la biomasa como la concentración máxima alcanzable en iguales condiciones de disponibilidad de nutrientes se vieron afectadas al alternar de configuración, disminuyendo ambos al operar en condiciones de flujo total debido a un menor acceso por parte de las células a la energía lumínica disponible.

En función de los valores obtenidos de velocidad de producción de biomasa para los flujos restringido y total respectivamente, el mayor volumen obtenido al cambiar de tipo de flujo atenta contra la captación de energía lumínica, provocando una disminución en la velocidad de crecimiento como así también en la concentración final máxima obtenible. Y si bien, de acuerdo a los resultados informados en la tabla 1, se han obtenido valores de producción de biomasa comparables e incluso algo mayores operando con el FBR en flujo total, sin conocer los costos de cultivo y separación de la biomasa pero sin duda mayores en este caso por mayor volumen de cultivo a procesar, se puede deducir que la configuración de flujo restringido aventajará significativamente a ésta en términos económicos.

Por su parte, el modelo matemático propuesto para la predicción de la evolución del cultivo durante la fase de crecimiento lineal de la biomasa ha mostrado ser de utilidad, toda vez que ha podido ser ajustado con los datos empíricos en forma conveniente en condiciones de media y alta concentración celular. El modelo se ha basado en la afectación de la velocidad específica máxima dentro de la ecuación cinética, con la relación entre irradiancia promedio en el interior del cultivo e irradiancia disponible en la superficie externa del mismo, tomando en cuenta de este modo el control que sobre el proceso provoca el ensombrecimiento entre células y toda vez que la irradiancia promedio se puede determinar en función de la concentración de biomasa. Se espera entonces que el modelo aquí propuesto pueda constituirse en una herramienta de utilidad para el diseño de fotobiorreactores industriales.

Finalmente, se hace consideración particular de que en la configuración de flujo total, la relación de volumen iluminado del FBR (la del espacio anular) a volumen total es de 0,64. Los valores de los cocientes de las velocidades de crecimiento o productividad lineal entre las variantes de flujo total y fraccionado para ambas especies cultivadas son sólo algo mayores $(0,72$ para $P$. tricornutum y 0,68 para Tetraselmis $s p$.) de acuerdo al modelo ajustado y prácticamente coincidentes en base a los resultados empíricos (0,67 y 0,64 respectivamente), lo que puede entenderse como una relación proporcional entre la velocidad de reproducción celular y la fracción del período de iluminación a que acceden las células en su recorrido por el interior del FBR (iluminación en el espacio anular y oscuridad en el tubo interno). Se entiende que esto respalda la suposición realizada a los efectos de aplicación del modelo propuesto con la configuración de flujo total, de que en el volumen de cultivo ubicado en el interior del cilindro interno del FBR se puede ignorar la captación de energía lumínica por parte de las células en condiciones de media y alta concentración.

\section{AGRADECIMIENTOS}

Se agradece el apoyo institucional y económico de la Secretaría de Ciencia y Técnica de la Universidad Nacional de la Patagonia San Juan Bosco.

\section{REFERENCIAS}

Acién, F.G., García, F., Sánchez, J.A., Fernández, J.M. y Molina, E., Modelling of biomass productivity in tubular photobioreactors for microalgal cultures: effects of dilution rate, tube diameter and solar irradiance, doi: 10.1002/(SICI)1097-0290(19980620)58:6<605::AID-BIT6>3.0.CO;2-M, Biotechnology and Bioengineering, 58, 6, 605-616 (1998)

Barbosa, M. J., Hoogakker, J. y Wijffels, R. H., Optimisation of cultivation parameters in photobioreactors for microalgae cultivation using the A-stat technique, doi:10.1016/S1389-0344(03)00033-9, Biomol. Eng., 20, 115$123(2003)$

Barbosa, M. J., Hadiyanto y Wijffels, R. H., Overcoming shear stress of microalgae cultures in sparged photobioreactors, doi: 10.1002/bit.10862, Biotechnology and Bioengineering, 85, 78-85 (2004)

Bernárdez, E. R., Claria, M. A. y Cassano, A. E., Analysis and design of photoreactors, Carberry and Varma (Eds.), Chemical Reaction and Reactor Design, Marcel Dekker, N. Y., EE.UU., 839-922 (1987) 
Evers, E. G., A model for light limited continuous cultures: growth, shading and maintenance, doi: 10.1002/bit.260380307, Biotechnology and Bioengineering, 38, 254-259 (1991)

García-Malea López, M. C., del Río Sánchez, E., Casas López, J. L., Acién Fernández, F.G., Fernández Sevilla, J.M., Rivas, J., Guerrero, M.G. y Molina Grima, E., Comparative analysis of the outdoor culture of Haematococcus pluvialis in tubular and bubble column photobioreactors, doi: 10.1016/j.jbiotec.2005.11.010, J. of Biotechnology, 123(3), 329-342 (2006)

Grobbelaar, J. U., Factors governing algal growth in photobioreactors: the "open" versus "closed" debate, doi: 10.1007/s10811-008-9365-x, J. Appl. Phycol., 21, 489-492 (2009)

Hosseini, N. S., Shang, H., Ross, G. M. y Scott, J. A., Microalgae cultivation in a novel top-lit gas-lift open bioreactor, doi: 10.1016/j.biortech.2015.05.092, Bioresource Technol., 192, $432-440$ (2015)

Joshi, J. B., Computational flow modelling and design of bubble column reactors, doi: 10.1016/S00092509(01)00273-1, Chem. Eng. Sci., 56(21), 5893-5933 (2001)

Kong, B. y Vigil, R. D., Simulation of photosynthetically active radiation distribution in algal photobioreactors using a multidimensional spectral radiation model, doi: 10.1016/j.biortech.2014.01.052, Bioresource Technol., 158, 141$148(2014)$

Kumar, K. y Das, D., Growth characteristics of Chorella sorokiniana in airlift and bubble column photobioreactors, doi: 10.1016/j.biortech.2012.03.074, Bioresource Technol., 116, 307-313 (2012)

Laing, I., Cultivation of Marine Unicelullar Algae, Laboratory Leaflet MAFF Direct. Fish. Res., Lowestoft, Inglaterra, 67, 1-31 (1991)

López Rosales, L., García Camacho, F., Sánchez Miron, A., Contreras Gómez, A. y Molina Grima, E., An optimisation approach for culturing shear-sensitive dinoflagellate microalgae in bench-scale bubble column photobioreactors, doi: 10.1016/j.biortech.2015.08.087, Bioresource Technol., 197, 375-382 (2015)

Luo, H-P. y Al-Dahhan, M. H., Analizing and Modeling of photobioreactors by combining first principles of physiology and hydrodynamics, doi: 10.1002/bit.10831, Biotechnology and Bioengineering, 85(4), 382-393 (2004)

Luo, H-P., Muthanna, H. y Al-Dahhan, M. H., Airlift column photobioreactors for Porphirydium sp. culturing: part I. Effects of hydrodynamics and reactor geometry, doi: 10.1002/bit.24361, Biotechnology and Bioengineering, 109(4), 932-941 (2012)

McLachlan, J., Growth media - marine, Handbook of Phycological Methods, ISBN 0521297478, 2a edición., 2551, Cambridge Univ. Press, Cambridge, Inglaterra (1973)

Merchuk, J. C., García Camacho, F. y Molina Grima, E., Photobioreactors - models of photosynthesis and related effects, Comprehensive Biotechnology, doi: 10.1016/B978-0-08-088504-9.09002-4, 2, 227-247, Elsevier, Amsterdam, Países Bajos (2011)

Michels, M. H., van der Goot, A. J., Norsker, N. H. y Wijffels, R. H., Effects of shear stress on the microalgae Chaetoceros muelleri, doi: 10.1007/s00449-010-0415-9, Bioprocess Biosyst. Eng., 33, 921-927 (2010)

Miron, A. S., Camacho, F. G., Gómez, A. C., Grima, E. M. y Chisti, Y., Comparative evaluation of compact photobioreactors for large-scale monoculture of microalgae, doi: 10.1016/S0168-1656(99)00079-6, J. Biotechnol., 70, 249-270 (1999)

Molina Grima, E., García Camacho, F., Sánchez Pérez, J. A., Fernández Sevilla, J. M., Acién Fernández, F. G. y Contreras Gómez, A., A mathematical model of microalgal growth in light-limited chemostat culture, doi: 10.1002/jctb.280610212, J. Chem. Tech. Bioeng., 61(2), 167-173 (1994)

Molina Grima, E., García-Camacho, F., Sánchez Pérez, J. A., Acién Fernández, F. G. y Fernández Sevilla, J. M., Evaluation of photosynthetic efficiency in microalgal cultures using average irradiance, Enzyme and Microbial Technology, 21(5), 375-381 (1997)

Molina Grima, E., Acién Fernández, F. G., García Camacho, F. y Chisti, Y., Photobioreactors: light regime, mass transfer and scaleup, J. Biotechnol., ISSN: 0168-1656, 70, 231-247 (1999) 
Pawar, S. B., Process engineering aspects of vertical column photobioreactors for mass production of microalgae, doi: 10.1002/cben.201600003, Chem. Bio. Eng. Rev., 3(3), 101-115 (2016)

Provasoli, L., Media and prospects for the cultivation of marine algae, Gap Soc. Plant Physical, 63-75 (1968)

Pruvost, J., Pottier, L. y Legrand, J., Numerical investigation of hydrodynamic and mixing conditions in a torus photobioreactor, doi: 10.1016/j.ces.2006.02.027, Chem. Eng. Sci., 61(14), 4476-4489 (2006)

Sánchez Mirón, A., Cerón García, M. C., García Camacho, F., Molina Grima, E. y Chisti, Y., Growth and biochemical characterization of microalgas biomass produced in bubble column and airlift photobioreactors: studies in fed-batch culture, doi: 10.1016/S0141-0229(02)00229-6, Enzyme and Microbial Technology, 31, 1015$1023(2002)$

Vial, C., Poncin, S., Wild, G. y Midoux, N., Experimental and theoretical analysis of the hydrodynamics in the riser of an external loop airlift reactor, doi: 10.1016/S0009-2509(02)00284-1, Chem. Eng. Sci., 57(22), 4745-4762 (2002)

Wu, X. y Merchuk, J. C., A model integrating fluid dynamics in the photosynthesis and photoinhibition process, doi: 10.1016/S0009-2509(01)00048-3, Chem. Eng. Sci., 56(11), 3527-3538 (2001)

Wu, X. y Merchuk, J. C., Simulation of algae grow/th in a bench scale internal loop airlift reactor, doi: 10.1016/j.ces.2004.02.019, Chem. Eng. Sci., 59(14), 2899-2912 (2004)

Xu, L., Weathers, P. J., Xiong, X. y Liu, C., Microalgal bioreactors: challenges and opportunities, doi: 10.1002/elsc.200800111, Eng. Life Sci., 9,178-189 (2009)

Ying, K., Gilmour, D. J., Shi, Y. y Zimmerman, W. B., Growth enhancement of Dunaliella salina by microbubble induced airlift bioreactor (ALB) - The relation beween mass transfer and growth rate, doi: 10.4236/jbnb.2013.42A001, J. Biomat. Nanobiotech., 4(2A), 1-9 (2013) 